\title{
LGRB hosts in emission and in absorption
}

\author{
Paul M. Vreeswijk \\ Department of Particle Physics and Astrophysics, \\ Weizmann Institute of Science, Rehovot 7610001, Israel \\ email: paul.vreeswijk@weizmann.ac.il
}

\begin{abstract}
Long-duration gamma-ray bursts (LGRBs) provide a unique way of selecting a sample of actively star-forming galaxies independent of their brightness and at practically any redshift. I will review what we know about the hosts and more immediate environments of LGRBs from two different perspectives: ultraviolet absorption-line spectroscopy of the bright early afterglow, and observations of their hosts in emission once the afterglow has faded away.
\end{abstract}

Keywords. Gamma rays: bursts - ISM: abundances - Galaxies: high-redshift

\section{Introduction}

There is strong evidence that long-duration gamma-ray bursts (LGRBs) originate from the explosion of a massive star (see Hjorth \& Bloom 2011, and references therein). LGRBs are extremely bright in gamma-rays, X-rays and the optical/near-infrared, making them observable at practically any redshift (so far up to $z=8.2$; Tanvir et al. 2009). This makes them excellent probes of massive star-forming regions at any redshift, independent of the amount of dust surrounding the explosion (at least in gamma- and X-rays) and independent of the host-galaxy brightness. The bright - but quickly fading - LGRB afterglow allows extraction of detailed information of the host-galaxy interstellar medium through ultraviolet (UV) absorption-line spectroscopy (see Sect. 2). After the afterglow has faded away, the host galaxy can be studied at leasure in emission (see Sect. 3). Through these two complementary perspectives, LGRBs can provide unique insight into the chemical evolution of (LGRB-selected) star-forming regions, the star-formation history of the universe, the distribution of $\mathrm{H}$ I gas, metals and dust surrounding the explosions and the gas kinematics, e.g. inflows and outflows, in actively star-forming galaxies.

\section{LGRB host galaxies in absorption}

Prompt UV absorption-line spectroscopy of LGRB afterglows show their host-galaxy interstellar medium to harbour large column densities of H I gas (e.g. Vreeswijk et al. 2004) and metals (e.g. Savaglio et al. 2003). The LGRB host metallicity as a function of redshift does not appear to evolve much (e.g. Savaglio et al. 2012); in any case its evolution is weaker than that inferred from quasar aborption-line studies. Only a very small fraction of LGRB sightlines show the presence of molecules in the host ISM (see Prochaska et al. 2009), but observations so far have been biased against their detection (see Krühler et al. 2013). The relatively simple broken power-law spectra of LGRB afterglows, from the optical to the X-ray regime, make them very suitable to probe dust extinction properties of the absorbing medium (e.g. Schady et al. 2007), even allowing the inference of an absolute extinction curve (Elíasdóttir et al. 2009). Most LGRB host extinction curves are consistent with an LMC type exctinction, but a non-negligible fraction shows the $2175 \AA$ A extinction bump prevalent along Milky Way sightlines (Zafar et al. 2011). Finally, detection of variable absorption of fine-structure lines of Fe II and Ni II have shown the 
absorbing neutral gas to be at least $100 \mathrm{pc}$ and up to kiloparsecs away from the LGRB explosion (e.g. Vreeswijk et al. 2007; D'Elia et al. 2009), even in the case where clear on-going ionisation is detected (Vreeswijk et al. 2013).

\section{LGRB host galaxies in emission}

In emission, the typical LGRB host is a faint, blue, low-mass galaxy (see Savaglio et al. 2009) with an irregular morphology (Fruchter et al. 2006) that is very actively forming stars and hence displaying prominent emission lines (e.g. Krühler et al. 2015). For a sample of low-redshift LGRBs, several different studies have found LGRBs to prefer low-metallicity environments (Modjaz et al. 2008). The metallicities of LGRB hosts fall below the local (SDSS) mass-metallicity relation (see Levesque et al. 2010), but they are consistent with the general population of galaxies when considering a more fundamental relation between mass, metallicty and star-formation rate (Mannucci et al. 2011).

The studies mentioned above are based on biased samples of LGRBs, whereas a signficant fraction $(\sim 25 \%)$ is "dark", i.e. not detected at optical wavelengths where most follow-up studies are being performed. It is important to include this missing population when making general inferences on the LGRB host-galaxy population. For example, Perley et al. (2013) find the hosts of a sample of dust-obscured LGRB afterglows to be much more massive, up to an order of magnitude, and to have higher star-formation rates than the hosts of non-obscured LGRBs. The very successful Swift mission has allowed the construction of such unbiased, complete samples, such as the following: TOUGH (Hjorth et al. 2012), GROND (Greiner et al. 2011), BAT6 (Vergani et al. 2015) and SHOALS (Perley et al. 2015). These are providing a more complete picture of LGRB hosts and are starting to addresss whether LGRBs are unbiased tracers of star formation.

\section{References}

D'Elia, V., Fiore, F., Perna, R., et al. 2009, ApJ, 694, 332

Elíasdóttir, Á., Fynbo, J. P. U., Hjorth, J., et al. 2009, ApJ, 697, 1725

Fruchter, A. S., Levan, A. J., Strolger, L., et al. 2006, Nature, 441, 463

Greiner, J., Krühler, T., Klose, S., et al. 2011, A\& A, 526, A30+

Hjorth, J. \& Bloom, J. S. 2011, arXiv:1104.2274

Hjorth, J., Malesani, D., Jakobsson, P., et al. 2012, ApJ, 756, 187

Krühler, T., Ledoux, C., Fynbo, J. P. U., et al. 2013, A\&A, 557, A18

Krühler, T., Malesani, D., Fynbo, J. P. U., et al. 2015, A\&A, 581, A125

Levesque, E. M., Kewley, L. J., Berger, E., \& Zahid, H. J. 2010, AJ, 140, 1557

Mannucci, F., Salvaterra, R., \& Campisi, M. A. 2011, MNRAS, 414, 1263

Modjaz, M., Kewley, L., Kirshner, R. P., et al. 2008, AJ, 135, 1136

Perley, D. A., Levan, A. J., Tanvir, N. R., et al. 2013, ApJ, 778, 128

Perley, D. A., Krühler, T., Schulze, S., et al. 2015, arXiv:1504.02482

Prochaska, J. X., Sheffer, Y., Perley, D. A., et al. 2009, ApJ, 691, L27

Savaglio, S., Fall, S. M., \& Fiore, F. 2003, ApJ, 585, 638

Savaglio, S., Glazebrook, K., \& Le Borgne, D. 2009, ApJ, 691, 182

Savaglio, S., Rau, A., Greiner, J., et al. 2012, MNRAS, 420, 627

Schady, P., Mason, K. O., Page, M. J., et al. 2007, MNRAS, 377, 273

Tanvir, N. R., Fox, D. B., Levan, A. J., et al. 2009, Nature, 461, 1254

Vergani, S. D., Salvaterra, R., Japelj, J., et al. 2015, A\&A A, 581, A102

Vreeswijk, P. M., Ellison, S. L., Ledoux, C., et al. 2004, A\&A A, 419, 927

Vreeswijk, P. M., Ledoux, C., Smette, A., et al. 2007, A\&A, 468, 83

Vreeswijk, P. M., Ledoux, C., Raassen, A. J. J., et al. 2013, AछA, 549, A22

Zafar, T., Watson, D., Fynbo, J. P. U., et al. 2011, A\&SA, 532, A143 